\title{
The use of the ENDF library for nucleosynthesis studies
}

\author{
Boris Pritychenko ${ }^{1, *}$ \\ ${ }^{1}$ National Nuclear Data Center, Brookhaven National Laboratory, Upton, NY 11973-5000, USA
}

\begin{abstract}
Stellar nucleosynthesis modeling studies would significantly benefit from the use of fully traceable, documented and unbiased nuclear data. The nuclear industry standard Evaluated Nuclear Data File (ENDF) libraries contain extensive collections of reaction data sets relevant to astrophysics. For the first time rapidand slow-neutron capture, $r$ - and $s$-process, respectively, abundances were computed from ENDF/B-VIII.0 and TENDL-2015 libraries and compared with available data. The current results highlight mutually beneficial relations between nuclear industry and nuclear astrophysics data developments.
\end{abstract}

\section{Introduction}

Recent multi-messenger detection of the binary neutron star merger (GW170817) and the indirect clues of $r$ process production of gold, platinum, and lanthanide elements [1-3] has generated tremendous new activity in stellar nucleosynthesis abundance calculations. These calculations strongly rely on astrophysical models and the quality of underlying nuclear data [4]. Previously the Karlsruhe Astrophysical Database of Nucleosynthesis in Stars (KADoNiS) [5] was extensively used in modeling of the slow neutron capture process ( $s$-process), and many KADoNIS 0.3 library cross sections were normalized (biased) to a ${ }^{197} \mathrm{Au}(\mathrm{n}, \gamma)$ activation measurement [6]. The activation Maxwellian-averaged cross section (MACS) 30 $\mathrm{keV}$ value of $582 \pm 9 \mathrm{mb}$ disagrees with the neutron nuclear cross section standard value of $620 \pm 11 \mathrm{mb}[7,8]$. Recently, the KADoNiS gold value has been re-examined with Monte Carlo simulations, extensive analysis of neutron time-of-flight (TOF), and activation experiments [9]. The comprehensive analysis of gold neutron capture cross sections showed an impact of neutron backing material scattering, while ENDF libraries are essentially based on the TOF-measurements and not affected by this issue. The revised ${ }^{197} \mathrm{Au}(\mathrm{n}, \gamma)$ activation MACS value of $612 \pm 6 \mathrm{mb}$ is consistent with the standard value, and the KADoNiS cross sections have been updated for 63 target nuclides from ${ }^{103} \mathrm{Rh}$ to ${ }^{197} \mathrm{Au}$ [9]. As of today, graphic representations of the corrected cross sections are publicly available, while numeric values will be disseminated later.

In the light of this disclosure, it would be advantageous to develop fully traceable, documented and unbiased nuclear data sets for stellar nucleosynthesis calculations. ENDF/B is a primary nuclear reaction data library for applications, and it is focused on target nuclei near the valley of stability. The ENDF/B library was originally produced by the Cross Section Evaluation Working Group (CSEWG) collaboration in 1968 for nuclear power plant

\footnotetext{
*e-mail: pritychenko@bnl.gov
}

design, criticality safety, shielding, and national security applications. It gained a worldwide popularity, many nations forged their own evaluated nuclear data libraries using the ENDF-6 format [10], and its user community has broadened into applied and fundamental sciences since introduction of evaluated neutron cross sections in MCNP, and GEANT computer codes simulations [11, 12]. Currently, the ENDF evaluations are conducted in partnership among the Organisation for Economic Co-operation and Development (OECD) and International Atomic Energy Agency (IAEA) organizations and member states $[13,14]$. The global partnership is responsible for neutron data evaluations for ENDF/B, JEFF (Joint Evaluated Fission and Fusion) File and TENDL (TALYS Evaluated Nuclear Data Library), JENDL (Japanese Evaluated Nuclear Data Library), ROSFOND (ROSsijsky File Otsenennykh Neutronnykh Dannykh, in Russian) and CENDL (Chinese Evaluated Nuclear Data Library) [15-20] in the United States, Western Europe/IAEA, Japan, Russian Federation, and China, respectively.

The recently released ENDF/B-VIII.0 library by the CSEWG collaboration [15] represents the state of the art in nuclear reaction data evaluations, and the library is briefly described below. It is a major nuclear reaction library that, for reactions that have been measured, includes evaluated (recommended) cross sections, spectra, angular distributions, fission product yields, thermal neutron scattering, photo-atomic, and decay data. Most of the reactions in ENDF are neutron-induced reactions. ENDF/B library evaluations are based on nuclear model predictions and normalized to experimental data, with an exception of neutron resonance region, where priority is directly given to experimental data. Its evaluations contain multiple sublibraries and complete data sets for a particular isotope or element. The neutron sublibrary covers all neutron reaction channels within the $10^{-5} \mathrm{eV}-20 \mathrm{MeV}$ energy range and includes comprehensive covariance files [21,22]. The covariance files are essential in uncertainty quantification 
processes driven by nuclear model and experimental user needs. An extensive CSEWG covariance development effort $[23,24]$ furnished multiple results.

The KADoNiS library has been used extensively for nuclear astrophysics calculations, while the ENDF libraries were largely overlooked. The initial attempt to calculate JENDL-3.3 library MACS and reaction rates [25] demonstrated an applicability of evaluated libraries for astrophysical tasks and, in some cases, the calculated values have deviated from KADoNiS values. These cross section discrepancies have been resolved in Brookhaven computations $[26,27]$ when MACS, reaction rates, and their uncertainties for multiple libraries have been produced and analyzed. The relevance of ENDF cross sections and reaction rates for stellar nucleosynthesis simulations have not yet been thoroughly examined. In this study, the applicability of evaluated neutron cross sections for $s$-process nucleosynthesis modeling are investigated using ENDF libraries, and solar $r$-process abundances are determined.

\section{Stellar Nucleosynthesis Modeling}

There are multiple nuclear astrophysics phenomena that are responsible for the present variety of elements and isotopes in the Universe [28], and pioneering works of E. Burbidge et al. and A. Cameron [29, 30] summarized the evidence for slow $(s)$ and rapid $(r)$ neutron capture processes. The $s$-process occurs in Asymptotic Giant Branch (AGB) and Red Giant stars, while the $r$-process likely occurs in neutron star mergers and core-collapse supernovae. Further analysis of the nuclear chart helps to identify exclusive $s-, r$-process, and mixed $(s+r)$ nuclei.

In this work, the classical model of stellar nucleosynthesis [31, 32] will be used to evaluate the data quality in evaluated libraries. The classical model is based on a phenomenological and site-independent approach, and it assumes that the seeds for neutron captures are made entirely of ${ }^{56} \mathrm{Fe}$. The $s$-process abundance of an isotope $N_{(A)}$ depends on its precursor $N_{(A-1)}$ quantity as in

$$
\frac{d N_{(A)}}{d t}=\lambda_{n(A-1)} N_{(A-1)}-\left[\lambda_{n(A)}+\lambda_{\beta(A)}\right] N_{(A)},
$$

where $\lambda_{n}$ is the neutron capture rate, and $\lambda_{\beta}=\frac{\ln 2}{T_{1 / 2}}$ is the $\beta$-decay rate for radioactive nuclei. Assuming that the temperature and neutron density are constant, and ignoring $s$ process branchings, the previous formula simplifies to

$$
\frac{d N_{(A)}}{d t}=\sigma_{(A-1)} N_{(A-1)}+\sigma_{(A)} N_{(A)} .
$$

Equation 2 was solved analytically for an exponential average flow of neutron exposure assuming that temperature remains constant over the whole timescale of the $s$ process [31]. The product of MACS and isotopic abundance $\left(\sigma_{(A)} N_{(A)}\right)$ was written as

$$
\sigma_{(A)} N_{(A)}=\frac{f N_{56}}{\tau_{0}} \prod_{i=56}^{A}\left[1+\frac{1}{\sigma(i) \tau_{0}}\right]^{-1},
$$

where $f$ and $\tau_{0}$ are the neutron fluence distribution parameters, and $N_{56}$ is the initial abundance of ${ }^{56} \mathrm{Fe}$ seed.
Maxwellian-averaged cross sections (MACS) in Eq. 3 are described as

$$
\sigma^{\operatorname{Maxw}}(k T)=\frac{2}{\sqrt{\pi}} \frac{a^{2}}{(k T)^{2}} \int_{0}^{\infty} \sigma\left(E_{n}^{L}\right) E_{n}^{L} e^{-\frac{a E_{n}^{L}}{k T}} d E_{n}^{L},
$$

where $a=m_{2} /\left(m_{1}+m_{2}\right), k$ and $T$ are the Boltzmann constant and temperature of the system, respectively, and $E$ is the energy of relative motion of the neutron with respect to the target $[4,25] . E_{n}^{L}$ is the neutron energy in the laboratory system, while $m_{1}$ and $m_{2}$ are the masses of the neutron and the target nucleus, respectively. Equation 4 has been used in the present work to calculate ENDF/BVIII.0 and TENDL-2015 MACS at $k T=30 \mathrm{keV}$. Prior to calculations the neutron resonance region evaluated data had been Doppler reconstructed at $\mathrm{T}=293.16 \mathrm{~K}$ with the PREPRO code [33]. These MACS were extensively used by the CSEWG collaboration during the ENDF/B-VIII.0 library validation process [15].

Analysis of solar system samples shows that $s$-process abundances originate from a superposition of the two major exponential distributions of time-integrated neutron exposure: weak component (responsible for the production of $70 \leq \mathrm{A} \leq 90$ nuclei), and the main component (for $90 \leq$ A $\leq 204$ nuclei). Further inquiry suggests a strong preference for the main $s$-process component fitting because of several issues in the $\mathrm{A}<90$ region such as lack of equilibrium [34], small number of $s$-process only medium nuclei and a limited number of measurements with these nuclei. Therefore, the strong component only is examined in the present work and nuclei abundances are taken from Ref. [35]. Neutron fluence parameters for $s$-process only isotopes were derived using Eq. 3 above. Later, the derived parameters were optimized using least squares procedures, and $f$ and $\tau_{0}$ neutron fluence distribution numerical values were obtained. The resulting fluence parameters are shown in Table 1.

Table 1. $S$-process strong component neutron fluence parameters for ENDF/B-VIII.0 and TENDL-2015 libraries $[15,16]$.

\begin{tabular}{l|c|c}
\hline \hline Parameters & ENDF/B-VIII.0 & TENDL-2015 \\
\hline $\mathrm{f}$ & $0.000434 \pm 0.000125$ & $0.000355 \pm 0.0000059$ \\
$\tau_{0}$ & $0.31256 \pm 0.03025$ & $0.37488 \pm 0.03013$ \\
\hline \hline
\end{tabular}

\section{Solar System Abundances}

$S$-process contribution to solar system abundances can be estimated and compared with observed values using neutron fluence parameters. The ENDF/B-VIII.0 MACS at $k T=30 \mathrm{keV}$ times abundance and $s$-process fitted product values are shown in Fig. 1. The Figure data indicate a surplus production for many nuclei compared with the $s$-process expectations. This surplus is commonly attributed to the $r$-process contribution, and this 'solar $r$ process abundance' is determined by subtracting the expected $s$-process production from the solar abundance for each isotope. 


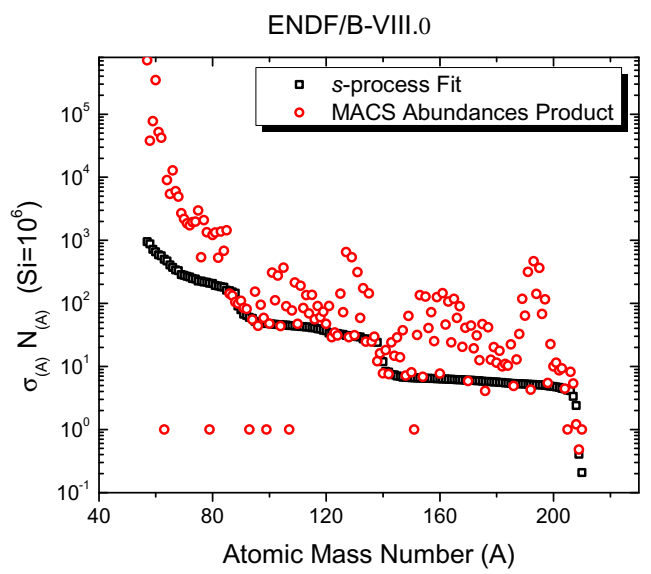

Figure 1. ENDF/B-VIII.0 neutron capture MACS at $k T=30 \mathrm{keV}$ times the solar system abundance and $s$-process fitted values as function of the atomic mass number. The solar system isotope abundances are taken from Ref. [35].

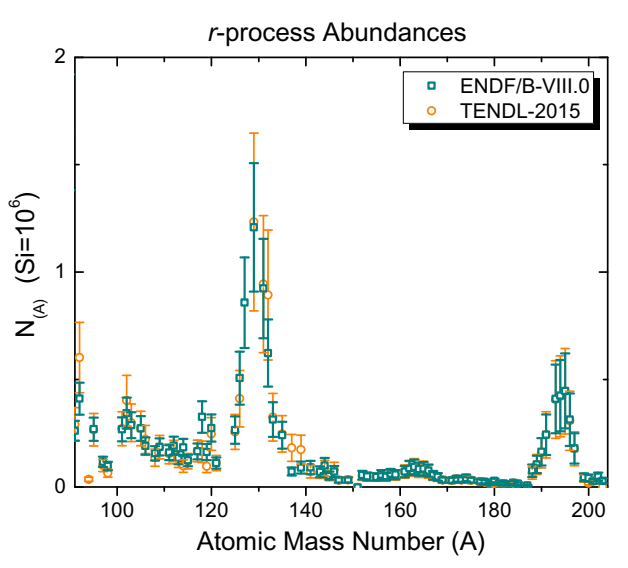

Figure 2. ENDF/B-VIII.0 and TENDL-2015 r-process abundances.

Solar $r$-process abundances determined via the ENDF/B-VIII.0 and TENDL-2015 libraries are shown in Fig. 2. A Fig. 2 data analysis shows two strong $r$-process abundance peaks and the broad surge due to production of lanthanides that were tentatively observed in neutron stars merger [1-3]. Shown in the Fig. 3 ENDF/B-VIII.0 $r$-process abundances agree well with solar system abundances that were deduced by M. Arnould et al. [36] from Ref. [37]. TENDL-2015 library produces similar values because several of its evaluations near the valley of stability are borrowed from other ENDF libraries. The current results demonstrate a large potential of evaluated libraries data for stellar nucleosynthesis calculations of certain scenarios and provide supplementary data for analysis of neutron star mergers as potential $r$-process astrophysical sites.

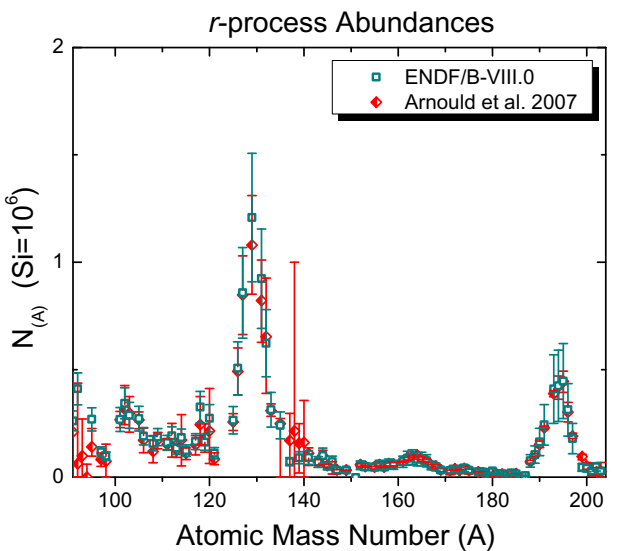

Figure 3. ENDF/B-VIII.0 and solar system $r$-process abundances that were deduced by M. Arnould et al. [36, 37].

\section{Conclusion}

In conclusion, the observations of $r$-process elements in neutron star mergers has generated tremendous new activity in stellar nucleosynthesis calculations and the corresponding nuclear data. Recent re-analysis of KADoNiS library [9] reveals multiple issues with the Karlsruhe data and a strong need for complementary data sets. The release of the ENDF/B-VIII.0 evaluated nuclear data library creates a unique opportunity for nuclear science and technology developments, and it is here used for nuclear astrophysics calculations. The Maxwellian-averaged cross sections and astrophysical reaction rates for $553 \mathrm{ENDF} / \mathrm{B}-$ VIII.0 and 2809 TENDL-2015 libraries target nuclides have been produced. These data were combined with the solar system abundances and fitted. Solar $r$-process abundances have been extracted in the present work, and an approximate agreement was shown with literature values.

The next stage of the current project will involve incorporation of the evaluated nuclear data libraries into astrophysical model codes. Work on ENDF/B-VIII.0 \& TENDL-2015 libraries data transfer for $(\mathrm{n}, \gamma),(\mathrm{n}, \alpha)$, $(\mathrm{n}, \mathrm{p})$ and (n,fission) reaction channels within 0.01-10 GK neutron temperatures into REACLIB formatted library $[38,39]$ is currently underway. The new complementary data sets will provide a valuable comparison to REACLIB reaction rates and will aid in efforts to continually improve the nuclear data that are the foundation of nucleosynthesis calculations.

The author is indebted to A. Sonzogni for encouragement of this project, D. Brown for useful comments, and V. Unferth and R. Arcilla for a careful reading of the manuscript and valuable suggestions. Work at Brookhaven was funded by the Office of Nuclear Physics, Office of Science of the U.S. Department of Energy, under Contract No. DE-AC02-98CH10886 with Brookhaven Science Associates, LLC. 


\section{References}

[1] E. Pian, P. D’Avanzo, S. Benetti, et al., NATURE(LoNDON) 551, 67 (2017).

[2] S.J. Smartt, T.-W. Chen, A. Jerkstrand, et al., NATURE(LONDON) 551, 75 (2017).

[3] N.R. Tanvir, A.J. Levan, C. Gonzalez-Fernandez, et al., Astrophys. J. 848, L27 (2017).

[4] C.E. Rolfs and W.S. Rodney, "Cauldrons in the Cosmos," The University of Chicago Press (1988).

[5] I. Dillmann, M. Heil, F. Käppeler, R. Plag, T. Rauscher, F-K. Thielemann, AIP Conference Proceedings 819, 123 (2006); Downloaded from 〈http://www.kadonis.org〉 on December 4, 2017.

[6] W. Ratynski, F. Käppeler, Phys. Rev. C 37, 595 (1988).

[7] A.D. Carlson, V.G. Pronyaev, R. Capote, et al., NucL. Data SheEts 123, 27 (2015).

[8] A.D. Carlson, V.G. Pronyaev, R. Capote, et al., NucL. Data SheEts 148, 143 (2018).

[9] R. Reifarth, P. Erbacher, S. Fiebiger, et al., Eur. Phys. J. Plus 133, 424 (2018).

[10] A. Trkov, M. Herman, D. A. Brown, "ENDF-6 Formats Manual," Brookhaven National Laboratory Report BNL-203218-2018-INRE, February 1, 2018.

[11] X-5 Monte Carlo Team, Los Alamos National Laboratory Report LAUR-03-1987 (2003). Available from 〈https://laws.lanl.gov/vhosts/mcnp.lanl.gov/pdf_files/laur-03-1987.pdf $\rangle$.

[12] J. Allison, K. Amako, J. Apostolakis et al., NucL. Instrum. Meth. Phys. Res. A 835, 186 (2016).

[13] Organisation for Economic Co-operation and Development (OECD), NEA-Data Bank. Available from $\langle$ https://www.oecd-nea.org/databank/〉.

[14] International Atomic Energy Agency (IAEA), NDS. Available from $\langle$ https://www-nds.iaea.org/ $\rangle$.

[15] D.A. Brown, M.B. Chadwick, R. Capote, et al., Nucl. Data Sheets 148, 1 (2018).

[16] A.J. Koning, D. Rochman, J. Kopecky et al., TENDL-2015: TALYS-based Evaluated Nuclear Data Library, Available from 〈https://tendl.web.psi.ch/tendl_2015/tend12015.html〉.

[17] A. J. Koning, E. Bauge, C.J. Dean et al., J. Korean Physical Society 59, No. 2, 1057 (2011).

[18] K. Shibata, T. Kawano, T. Nakagawa et al., J. NucL. Science and Technology 48, 1 (2011).

[19] S.V. Zabrodskaya, A.V. Ignatyuk, V.N. Koscheev et al., VANT, Nuclear Constants 1-2, 3 (2007).
[20] Z.G. Ge, Z.X. Zhao, H.H. Xia et al., J. Korean Physical Society 59 (2), 1052 (2011).

[21] D.L. Smith, Nucl. Data SheEts 109, 2915 (2008).

[22] D.A. Brown, M. Herman, S. Hoblit et al., J. Phys G 42, 034020 (2015).

[23] R.C. Little, T. Kawano, G.D. Hale et al., Nucl. Data SHEETs 109, 2828 (2008).

[24] S. Hoblit, Y.-S. Cho, M. Herman et al., Nucl. Data SHEETs 112, 3075 (2011).

[25] T. Nakagawa, S. Chiba, T. Hayakawa, T. Kajino, Aт. Data Nucl. Data Tables 91, 77 (2005).

[26] B. Pritychenko, S.F. Mughabghab, A.A. Sonzogni, At. Data Nucl. Data Tables 96, 645 (2010).

[27] B. Pritychenko, S.F. Mughabghab, Nucl. DatA SHEETs 113, 3120 (2012).

[28] R.N. Boyd, "An Introduction to Nuclear Astrophysics," The University of Chicago Press (2008).

[29] E.M. Burbidge, G.R. Burbidge, W.A. Fowler, F. Hoyle, Rev. Mod. Phys. 29, 547 (1957).

[30] A.G.W. Cameron, "Stellar Evolution, Nuclear Astrophysics and Nucleogenesis," AECL-454, CRL-41 (1957).

[31] D.A. Clayton, R.A. Ward, Astrophys. Journal 193, 397 (1974).

[32] F. Käppeler, H. Beer, K. Wisshak, D.D. Clayton, R.L. Macklin, R.A. Ward, Astrophys. Journal 257, 821 (1982).

[33] D.E. Cullen, International Atomic Energy Agency Report IAEA-NDS-39 (2015); Rev. 16, January 31, 2015. Available from 〈https://wwwnds.iaea.org/public/endf/prepro/ $>$.

[34] M. Lugaro, Journal of Physics: Conference Series 703, 012003 (2016).

[35] E. Anders, N. Grevesse, Geochim. Cosmochim. Act. 53, 197 (1989).

[36] M. Arnould, S. Goriely, K. Takahashi, Phys. RePt. 450, 97 (2007).

[37] H. Palme, H. Beer, Abundances of the Elements in the Solar System, in Landolt Börnstein, New Series, Group VI, Astron. \& Astrophys., Vol. 3, Subvol. a, (Berlin: Springer), p. 196 (1993); https://materials.springer.com/lb/docs/sm_lbs_9783-540-31380-9_56.

[38] T. Rauscher, F.-K. Thielemann, At. Data Nucl. Data TABLES 75, 1 (2000).

[39] T. Rauscher, F.-K. Thielemann, At. Data Nucl. Data TABLES 79, 47 (2001). 